\title{
Effect of time delay on vibroimpact dynamics in a forced Hertzian contact oscillator
}

\author{
L. Mokni, A. Bichri, M. Belhaq \\ Laboratory of Mechanics, University Hassan II-Casablanca, Morocco
}

\begin{abstract}
Vibroimpact dynamics of a forced single-sided Hertzian contact oscillator are typically initiated by jumps phenomena near resonances. It was shown that high-frequency excitations can be used to shift the threshold of such vibroimpacts toward higher or lower frequencies of the basic excitation depending on the type of the high-frequency excitation. These excitations not only produce a shift, but also soften the system behavior. The present paper investigates the effect of time delay state feedback on the threshold of vibroimpact dynamics of a forced Hertzian contact oscillator near the primary resonance. It is shown that for certain combinations of the gain parameters of delay state feedback, the jumps disappear leading the system to be maintained in contact when the excitation frequency sweeps through the resonance.
\end{abstract}

\section{Introduction}

In machinery in general and in rotating equipment in particular, many mechanisms ensuring transformation of motions typically use Hertzian contacts, as in ball or roller bearing, gears, push-cam systems, to quote a few; ses for instance [1-5] and references therein. Usually, a single-degree-of-freedom system is considered for modelling the behavior of a moving surface under a Hertzian contact. The performance of mechanisms evolving in such a contact-mode in term of preventing high levels of vibration, noise and contact loss, requires the contact-mode regime to be maintained during a scan of decreasing or increasing frequency through resonances. This wanted dynamic phenomenon has been studied for an idealized preloaded and nonsliding dry Hertzian contact near resonances using numerical, analytical and experimental approches [68]. It was concluded that the loss of contact is typically initiated by jumps near the resonances. Therefore, performing techniques for controlling the location of jumps is highly essential in order to enhance the performance of the system and to prevent its deterioration. In this context, three strategies were proposed to control the jumps in a Hertzian contact oscillator near the primary resonance [9]. The first strategy adds a fast harmonic excitation to the basic harmonic force from above, the second one uses a fast harmonic base displacement, while the third strategy considers a fast harmonic and parametric stiffness. It was shown that the excitation from above or through base displacement shifts the resonance curve left, whereas the fast parametric stiffness shifts the response curve right. A recent study was carried out near sub- and superharmonic resonances of order 2 and similar phenomena were found [10]. The results demonstrated principally that such control techniques produce a shift and softens the system behavior.
In this paper, the effect of time delay state feedback on the vibroimpact threshold of a forced Hertzian contact oscillator is analyzed near the primary resonance. The time delay is introduced in displacement and in velocity. The purpose is to examine whether the loss of contact phenomenon can be controlled when the delay is introduced either in the displacement, or in the velocity, or in both.

It is worth noticing that previous works investigated the dynamic near the primary resonance under time delay effect. For instance, the primary resonance of a Duffing oscillator subjected to both delayed position and velocity was studied in [11], and the case where two time delays are introduced in the state feedback has been analyzed in $[12,13]$. A study on the frequency island in the delayed primary resonance was carried out in [14]. Other works analyzed various nonlinear phenomena in delayed van der Pol equation, including control of limit cycle, can be found in [15$22]$.

In contrast to all these studies which are restricted to Duffing-Van der Pol systems, the present paper focuses on the investigation of vibroimpact triggering in a forced contact oscillator in which the nonlinear restoring force is of Hertzian type. Our motivation in this paper is to take advantage of the effective damping characteristic introduced by time delay to control or eliminate the birth of vibroimpact in the system under consideration.

The paper is organized as follows. The next section presents the equation of motion, performs the multiple scale method near the primary resonance and provides the frequency-response equation. In Section 3, the influence of time delay state feedback on the loss of contact is investigated for different cases of delay state feedback. Numerical simulations are conducted in each case of study to validate the analytical predictions. Section 4 concludes the work. 


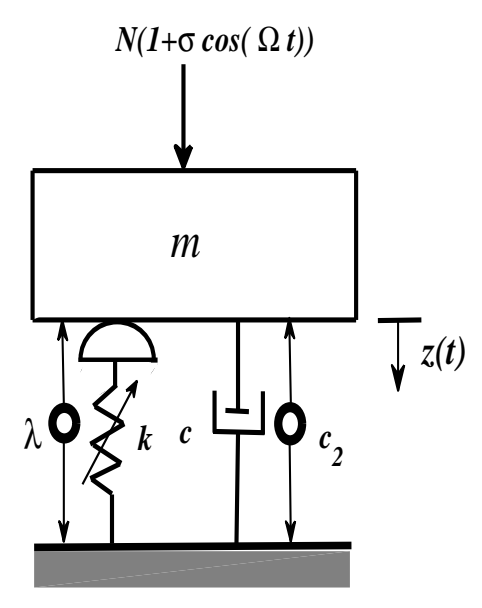

Fig. 1. Schematic of the dynamic model

\section{Equation of motion and frequency-response}

Consider a vertically forced single-sided Hertzian contact oscillator with delayed feedback in position and velocity modelled by a single degree-of-freedom system such that the moving mass $m$ is supported by a nonlinear restoring force [23]. A schematic model is shown in Fig. 1. The equation of motion can be written in the form $[7,9,10]$

$$
m \ddot{z}+c_{1} \dot{z}+k z^{\frac{3}{2}}=N(1+\sigma \cos \Omega t)+\lambda z_{T^{*}}+c_{2} \dot{z}_{T^{*}}
$$

where $z_{T^{*}}=z\left(t-T^{*}\right)$ and $\dot{z}_{T^{*}}=\dot{z}\left(t-T^{*}\right)$. Here $z$ is the normal displacement of the rigid mass, $c_{1}$ the damping coefficient, $k$ the constant given by the Hertzian theory [23], $N$ the static normal load, $\sigma$ and $\Omega$ are the level of the excitation and its frequency, respectively, while $\lambda$ and $c_{2}$ denote the gains of the delayed states, and $T^{*}$ is the time delay. The static contact compression $z_{s}$ is given by $z_{s}=\left(\frac{N}{k}\right)^{\frac{2}{3}}$. Introducing the variable changes as $\omega=\left(\frac{\Omega}{v}\right), v^{2}=\left(\frac{3 k}{2 m}\right) z_{s}^{\frac{1}{2}}, \eta=\frac{\lambda}{m v^{2}}, \xi_{1}=\frac{c_{1}}{2 m v}, \xi_{2}=\frac{c_{2}}{2 m v}$, $x=\frac{3\left(z-z_{s}\right)}{2 z_{s}}, \tau=v t$ and $T=v T^{*}$, the dimensionless equation of motion takes the form

$$
\begin{aligned}
x^{\prime \prime}+2 \xi_{1} x^{\prime}+\left(1+\frac{2}{3} x\right)^{\frac{3}{2}}= & 1+\sigma \cos \omega \tau+\eta x(\tau-T)+ \\
& 2 \xi_{2} x^{\prime}(\tau-T)+\frac{3}{2} \eta
\end{aligned}
$$

where the prime denotes deffirentiation with respect to $\tau$.

Expanding the nonlinear restoring force in Taylor series around the static load and neglecting terms of order greater than three in $x$, Eq. (2) takes the form

$$
\begin{aligned}
x^{\prime \prime}+\alpha_{1} x^{\prime}+\omega_{0}^{2} x+\beta x^{2}-\gamma x^{3}= & \sigma \cos \omega \tau+\eta x(\tau-T)+ \\
& \alpha_{2} x^{\prime}(\tau-T)+\frac{3}{2} \eta
\end{aligned}
$$

where $\alpha_{1}=2 \xi_{1}, \alpha_{2}=2 \xi_{2}, \beta=\frac{1}{6}, \gamma=\frac{1}{54}, \omega_{0}^{2}=1$ and $G=-\frac{3}{2} \eta$. To analyse the dynamic near the primary resonance, we express the resonance condition by introducing a detuning parameter $\delta$ according to

$$
\omega_{0}^{2}=\omega^{2}+\delta
$$

Introducing a small bookkeeping parameter $\mu$ and scaling the parameters in Eq. (3) as $\alpha_{1}=\mu \alpha_{1}, \alpha_{2}=\mu \alpha_{2}$, $G=\mu G, \beta=\mu \beta, \sigma=\mu \sigma, \delta=\mu \delta$ and $\gamma=\mu^{2} \gamma$, yields

$$
\begin{aligned}
x^{\prime \prime}+\omega^{2} x & =\mu\left(-\alpha_{1} x^{\prime}-\beta x^{2}+\sigma \cos \omega \tau-\delta x\right. \\
& \left.+\eta x(\tau-T)+\alpha_{2} x^{\prime}(\tau-T)-G\right)+\mu^{2} \gamma x^{3}
\end{aligned}
$$

Using the multiple scales method [24], we obtain the slow flow modulation equation of amplitude and phase

$$
\left\{\begin{array}{l}
\frac{d r}{d \tau}=A r+H_{1} \sin \theta+H_{2} \cos \theta \\
r \frac{d \theta}{d \tau}=B r+C r^{3}+H_{1} \cos \theta-H_{2} \sin \theta
\end{array}\right.
$$

where $A=-\left(\frac{\alpha_{1}}{2}+\frac{\eta}{\omega} \sin (\phi)-\frac{\alpha_{2}}{2} \cos (\phi)\right), B=\frac{\delta}{2 \omega}-\frac{\alpha_{2}}{2} \sin (\phi)-$ $\frac{\eta}{2 \omega} \cos (\phi)+\left(\frac{\alpha_{2} \delta-\eta \alpha_{1}}{4 \omega^{2}}\right) \sin (\phi)+\left(\frac{\alpha_{2} \alpha_{1} \omega^{2}+\eta \delta}{4 \omega^{3}}\right) \cos (\phi)-\left(\frac{\eta^{2}+\delta^{2}}{8 \omega^{3}}\right)-$ $\left(\frac{\alpha_{1}^{2}+\alpha_{2}^{2}}{8 \omega}\right)-\frac{\beta G}{\omega^{3}}, C=-\left(\frac{5 \beta^{2}}{12 \omega^{3}}+\frac{3 \gamma}{8 \omega}\right), H_{1}=\frac{\delta \sigma}{8 \omega^{3}}-\frac{\sigma}{2 \omega}-F_{1} \cos (\phi)-$ $F_{2} \sin (\phi), H_{2}=\frac{\alpha_{2} \sigma}{8 \omega^{2}}+F_{1} \sin (\phi)-F_{2} \cos (\phi), F_{1}=\frac{\sigma \eta}{8 \omega^{3}}$, $F_{2}=\frac{\sigma \alpha_{2}}{8 \omega^{2}}$ and $\phi=\omega T$. Eliminating the phase $\theta$ from the system (6), we obtain the following amplitudefrequency response consisting of a six order algebraic equation of the amplitude $r$

$$
C^{2} r^{6}+2 B C r^{4}+\left(A^{2}+B^{2}\right) r^{2}-\left(H_{1}^{2}+H_{2}^{2}\right)=0
$$

\section{Influence of delay states on the frequency-response}

In this section, we analyze the effect of delayed displacement and delayed velocity on the frequency-response and on jumps phenomena. Hereafter, we fix the parameters $\sigma=0.04$ and $\xi_{1}=0.01$. Figure 2 illustrates the frequency-response curves, as given by Eq. (7), in the case where the delayed feedback is present only in the position $\left(\eta \neq 0, \xi_{2}=0\right)$, for the time delay $T=0.1$.

In Fig. 3, we show also the frequency-response in the case where the delayed feedback is introduced in the position $\left(\eta \neq 0, \xi_{2}=0\right)$, and for the time delay $T=3$. It is clearly seen from these figures that for a small increase of $\eta$, the response curve shifts toward higher frequencies and the softening characteristic decreases slightly.

In Fig. 4 is shown the frequency-response curves, as given by Eq. (7), in the case of delayed velocity $(\eta=$ $0, \xi_{2} \neq 0$ ), and for the time delay $T=3$. The plots indicate that as the gain of the delayed velocity increases slightly, the peak amplitude in the frequency-response decreases, and the jumps are suppressed (Fig. 4c).

Figures 5 and 6 depict the effect of the feedback gains $\eta$ and $\xi_{2}$ when applied simultaneously. The plots show that increasing $\eta$ and $\xi_{2}$ simultaneously causes the peak amplitude of the response to decrease, and the 

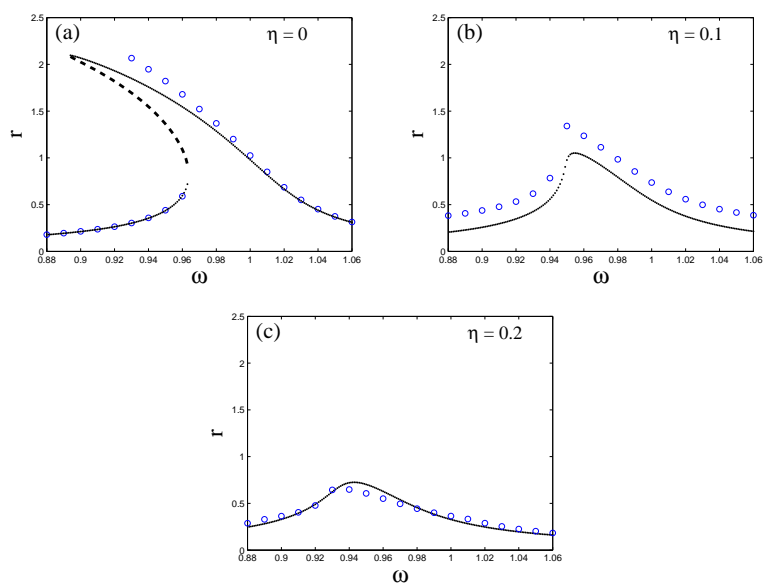

Fig. 2. Frequency-response curves versus $\omega$ for $T=0.1$ and $\xi_{2}=$ 0
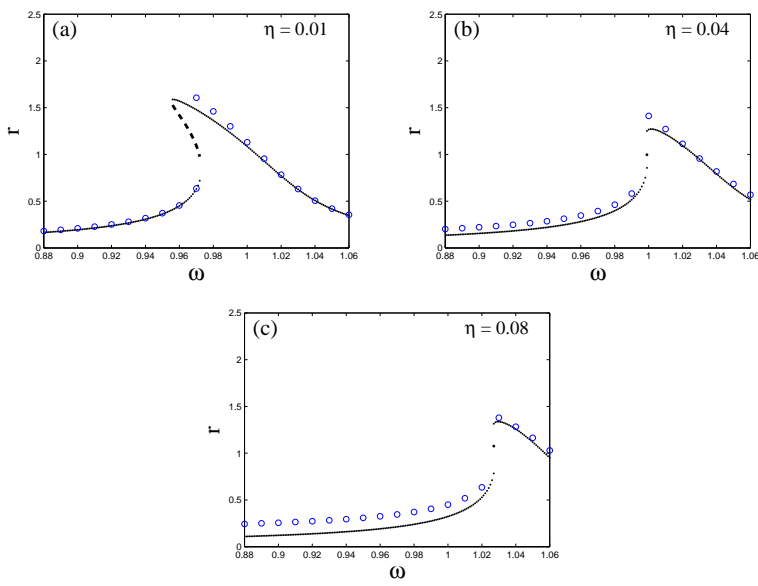

Fig. 3. Frequency-response curves versus $\omega$ for $T=3$ and $\xi_{2}=0$.
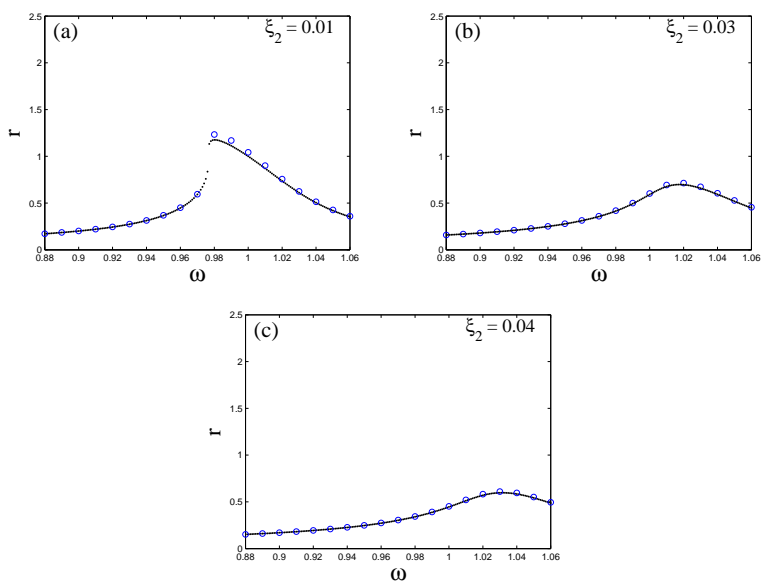

Fig. 4. Frequency-response curves versus $\omega$ for $T=4$ and $\eta=0$.

jump to disappear. Also, by inspecting these figures, it is evident that, for the same values of $\eta$ and $\xi_{2}$, a decrease of time delay from $T=3$ to $T=1$ causes the peak of the amplitude to decrease. This finding can be understood in Fig. 7.

Indeed, Fig. 7 depicts the variation of the amplitude versus the time delay $T$ for different values of the displacement gain $\eta$ (Fig. 7a, with $\left.\xi_{2}=0\right)$, and of the velocity gain $\xi_{2}$ (Fig. $7 \mathrm{~b}$, with $\eta=0$ ). These fig-
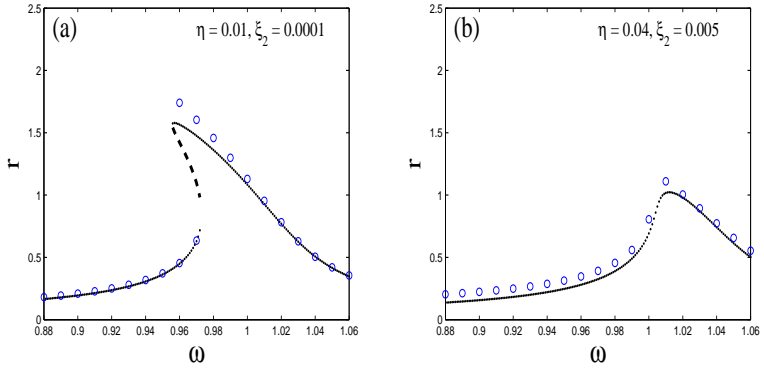

Fig. 5. Frequency-response curves versus $\omega$ for $T=3$.
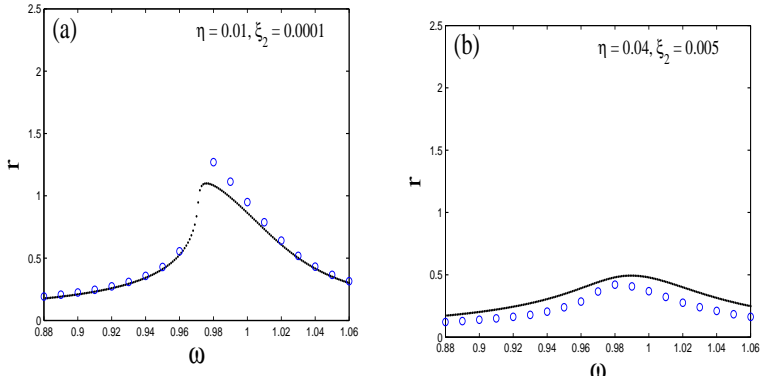

Fig. 6. Frequency-response curves versus $\omega$ for $T=1$.
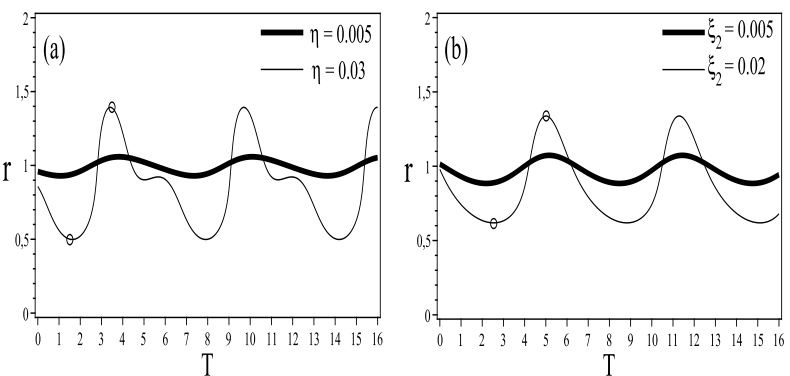

Fig. 7. Amplitude variation versus $T$ for $w=1$. (a): $\xi_{2}=0$; (b): $\eta=0$.
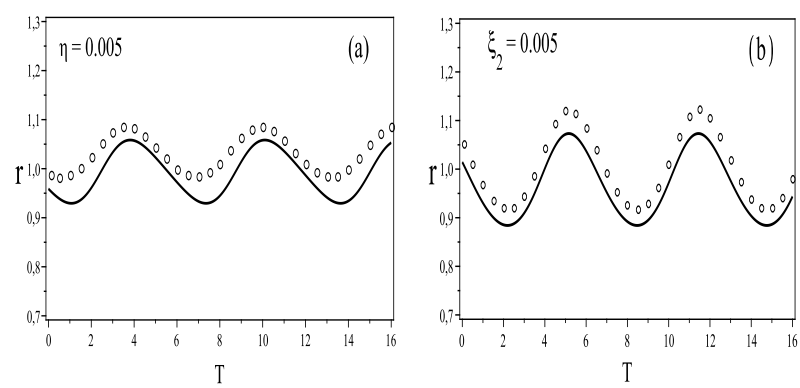

Fig. 8. Amplitude variation versus $T$ for $w=1$. Analytic (solid line), Numeric (circles). (a): $\xi_{2}=0 ;$ (b): $\eta=0$.

ures show the periodic characteristic of the amplitude variation with respect to time delay $T$.

To validate this analytical finding, Fig. 8 compares the amplitude variation obtained analytically (solid line) and numerically (circles) by using the fourthorder Runge-Kutta method.

Finally, in Fig. 9 is shown the frequency-response curves for two different values of $T$ picked from Fig. 7 and corresponding to minimum and maximum points 

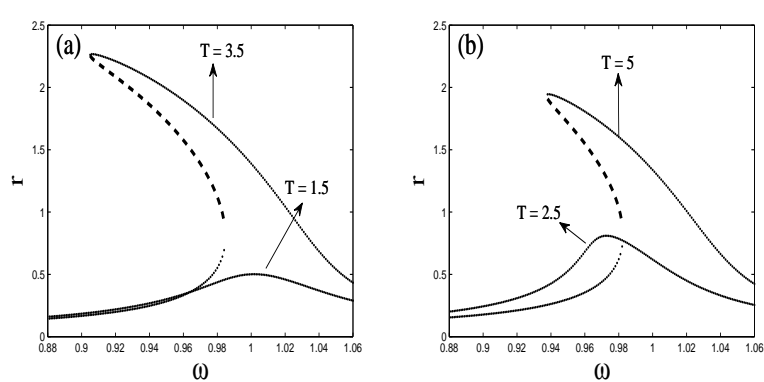

Fig. 9. Frequency-response curves versus $w$. (a): $\eta=0.03, \xi_{2}=0$; (b) for $\eta=0, \xi_{2}=0.02$.

of the amplitude response. points are indicated on the curves (circles).

\subsection{Conclusion}

The influence of time delay state feedback on the vibroimpact threshold of a Hertzian contact oscillator has been investigated near the primary resonance. The delay was introduced in the position and in the velocity. The method of multiple scales was used and the amplitude-frequency relations were derived. It was shown that a slight increase of the gain parameters, when applied separately or simultaneously, produces a shift of the amplitude-frequency response and suppresses jumps phenomena, ensuring the system to be maintained in contact when the excitation frequency sweeps through the resonance. The analytical prediction of the amplitude variation summarizes the possible situations of the frequency-response when the time delay is varied. Specifically, for some gain combinations, the peak amplitude undergoes a periodic variation, such that the peak decreases in certain intervals eliminating softening and jumps behavior, and increases in others producing softening and jumps in the system.

\section{References}

1. R. Nayak, Contact vibrations, J. Sound Vib, 22, (1972) 297-322.

2. D. Hess, A. Soom, Normal vibrations and friction under harmonic loads: Part 1-Hertzian contact. ASME Journal of Tribology, 113, (1991)80-86.

3. J. Sabot, P. Krempf, C. Janolin, Nonlinear vibrations of a sphere-plane contact excited by a normal load. J Sound Vib 214, (1998)359-375.

4. R. Carson, K. Johnson, Surface corrugations spontaneously generated in a rolling contact disc machine., 17 Wear (1971) 59-72.

5. A. Soom, JW. Chen, Simulation of random surface roughness-induced contact vibrations at Hertzian contacts during steady sliding. ASME Journal of Tribology, 108, (1986) 123-127.

6. E. Rigaud, P. Perret-Liaudet, Experiments and numerical results on nonlinear vibrations of an impacting hertzian contact. part 1: harmonic excitation. J Sound Vib, 265, (2003)289-307.
7. J. Perret-Liaudet, E. Rigaud, Response of an impacting Hertzian contact to an order-2 subharmonic excitation : Theory and experiments, J Sound Vib, 296, (2003) 319-333.

8. J. Perret-Liaudet, E. Rigaud, Superharmonic resonance of order 2 for an impacting Hertzian contact oscillator: Theory and experiments., J Comput Nonlinear Dyn, 2, (2007) 190-196.

9. A. Bichri, M. Belhaq, J. Perret-Liaudet, Control of vibroimpact dynamic of a single-sided-Hertzian contact forced oscillator. Nonlinear Dyn, 63, (2011) 51-60.

10. A. Bichri, M.Belhaq, Control of a forced impacting Hertzian contact oscillator near sub- and superharmonics of order 2. J Comput Nonlinear Dyn, Issue date: Jan (2012);7(1).

11. H. Hu, EH. Dowell, L. Virgin, Resonances of a harmonically forced duffing oscillator with time delay state feedback. Nonlinear Dyn, 15, (1998) 311-327.

12. Ji. JC, AYT.Leung, Resonances of a nonlinear SDOF system with two time-delays in linear feedback control. Nonlinear Dyn, (2002) 253, 985.

13. Y. Jin, H. Hu, Primary resonance of a duffing oscillator with two distinct time delays in state feedback under narrow-Bband random excitation. In: Proceedings of the ASME (2007), International Design and Engineering Technical Conference And Computers and Information in Engineering Conference. Las Vegas; NV (2007).

14. MF. Daqaq, GW. Vogl, Frequency island in the primary resonance of nonlinear delay systems. ENOC (2008), Saint Petersburg, Russia; June, 30July, 4 (2008).

15. FM. Atay, Van der Pol's oscillator under delayed feedback. J Sound Vib, 218, (1998) 333-339.

16. A. Maccari, Vibration control for the primary resonance of the van der Pol oscillator by a time delay state feedback., Int J Non-Linear Mech, 38, (2003)123-131.

17. A. Maccari, Delayed feedback control for a parametrically excited van der Pol oscillator., Physica Scripta , 76, (2007) 526-532.

18. AF. EI-Bassiouny, Stability and oscillation of two coupled Duffing equations with time delay state feedback., Physica Scripta, 74, (2006) 726-35.

19. SM. Sah, M. Belhaq, Effect of vertical highfrequency parametric excitation on self-excited motion in a delayed van der Pol oscillator. Chaos, Solitons \& Fractals, 37, (2008) 1489-1496.

20. M. Belhaq, SM. Sah Fast parametrically excited van der Pol oscillator with time delay state feedback.,Int J Non-linear Mech, 34, (2008) 124-130.

21. M. Belhaq, SM. Sah, Horizontal fast excitation in delayed van der Pol oscillator., Commun Nonlinear Sci Numer Simul, 13, (2008)1706-1713.

22. MK. Suchorsky, MS. Sah, RH. Rand, Using delay to quench undesirable vibrations, Nonlinear Dyn, 62, (2010) 407-416.

23. KL. Johnson, Contact Mechanics. Cambridge University Press: Cambridge; (1979).

24. AH. Nayfeh, DT. Mook, Nonlinear Oscillations. Wiley, New York; (1979). 